\title{
Modelling the radio pulses of an ultracool dwarf
}

\author{
S. Yu ${ }^{1}$, G. Hallinan ${ }^{2,3}$, J. G. Doyle ${ }^{1}$, A. L. MacKinnon ${ }^{4}$, A. Antonova ${ }^{5}$, A. Kuznetsov ${ }^{1,6}$, A. Golden ${ }^{2}$, and Z. H. Zhang ${ }^{7}$ \\ 1 Armagh Observatory, College Hill, Armagh BT61 9DG, N. Ireland \\ e-mail: syu@arm.ac.uk \\ 2 Centre for Astronomy, National University of Ireland, Galway, Ireland \\ 3 Department of Astronomy, University of California, Berkeley, CA 94720-3411, USA \\ ${ }^{4}$ Department of Adult and Continuing Education, University of Glasgow, Scotland \\ 5 Department of Astronomy, Faculty of Physics, St. Kliment Ohridski, University of Sofia, Bulgaria \\ ${ }^{6}$ Institute of Solar-Terrestrial Physics, Irkutsk 664033, Russia \\ 7 Centre for Astrophysics Research, Science and Technology Research Institute, University of Hertfordshrie, Hatfield AL10 9AB
}

Received 13 August 2010 / Accepted 8 September 2010

\section{ABSTRACT}

\begin{abstract}
Context. Recently, unanticipated magnetic activity in ultracool dwarfs (UCDs, spectral classes later than M7) has emerged from a number of radio observations. The highly (up to $100 \%$ ) circularly polarized nature and high brightness temperature of the emission have been interpreted as requiring an effective amplification mechanism of the high-frequency electromagnetic waves - the electron cyclotron maser instability (ECMI).

Aims. We aim to understand the magnetic topology and the properties of the radio emitting region and associated plasmas in these ultracool dwarfs, interpreting the origin of radio pulses and their radiation mechanism.

Methods. An active region model was built, based on the rotation of the UCD and the ECMI mechanism.

Results. The high degree of variability in the brightness and the diverse profile of pulses can be interpreted in terms of a large-scale hot active region with extended magnetic structure existing in the magnetosphere of TVLM 513-46546. We suggest the time profile of the radio light curve is in the form of power law in the model. Combining the analysis of the data and our simulation, we can determine the loss-cone electrons have a density in the range of $1.25 \times 10^{5}-5 \times 10^{5} \mathrm{~cm}^{-3}$ and temperature between $10^{7}$ and $5 \times 10^{7} \mathrm{~K}$. The active region has a size $<1 R_{\text {Jup }}$, while the pulses produced by the ECMI mechanism are from a much more compact region (e.g. $\sim 0.007 R_{\text {Jup }}$ ). A surface magnetic field strength of $\approx 7000 \mathrm{G}$ is predicted.

Conclusions. The active region model is applied to the radio emission from TVLM 513-46546, in which the ECMI mechanism is responsible for the radio bursts from the magnetic tubes and the rotation of the dwarf can modulate the integral of flux with respect to time. The radio emitting region consists of complicated substructures. With this model, we can determine the nature (e.g. size, temperature, density) of the radio emitting region and plasma. The magnetic topology can also be constrained. We compare our predicted X-ray flux with Chandra X-ray observation of TVLM 513-46546. Although the X-ray detection is only marginally significant, our predicted flux is significantly lower than the observed flux. Further multi-wavelength observations will help us better understand the magnetic field structure and plasma behavior on the ultracool dwarf.
\end{abstract}

Key words. magnetic fields - radio continuum: stars - stars: low-mass - brown dwarfs - polarization - masers

\section{Introduction}

A large number of recent radio observations indicate that intense magnetic activity exists in ultracool dwarfs (UCDs), i.e. objects with spectral type later than M7. Berger et al. (2001) reported the first detection of quiescent and flaring radio emission from the M9 brown dwarf LP944-20, in which a bright X-ray flare was also detected (Rutledge et al. 2000), with anomalous quiescent radio luminosity at least four orders of magnitude larger than predicted from an empirical relation between the X-ray and radio luminosities of active stars with spectral types from $\mathrm{F}$ to M (Guedel \& Benz 1993). The detection of electron cyclotron maser (ECM) emission provided the first confirmation of kilogauss fields for a late M dwarf (Hallinan et al. 2006), and subsequently led to the discvery that even cooler L type dwarfs can also possess magnetic fields in the kilogauss range (Hallinan et al. 2008). Radio observations exclusively enable the measurement of magnetic fields on cool, brown dwarfs, and possibly also for the very faint exoplanets (Zarka 2007).

Chromospheric $\mathrm{H} \alpha$ emission and coronal X-ray emission show a sharp decline in $L_{\mathrm{H} \alpha} / L_{\mathrm{bol}}$ and $L_{\mathrm{X}} / L_{\text {bol }}$ beyond spectral type M7 (Neuhäuser et al. 1999; Gizis et al. 2000
West et al. 2004; Stelzer et al. 2006a; Schmidt et al. 2007) which would be consistent with lower fractional ionization in atmospheres of later spectral type (Mohanty et al. 2002). Recently, however, a lot of evidence, such as quiescent and flaring $\mathrm{H} \alpha$ emission from some $\mathrm{L}$ and $\mathrm{T}$ dwarfs (Reid et al. 1999; Burgasser et al. 2000; Liebert et al. 2003; Reiners \& Basri 2007; Rockenfeller et al. 2006; Stelzer et al. 2006b; Schmidt et al. 2007), FeH lines from cool M dwarfs (Afram et al. 2009), strong X-ray emission from one such source (Audard et al. 2007), all suggest that perhaps an efficient magnetic dynamo could be operational (Parker 1955) in a fraction of the UCD population (Reiners \& Basri 2010) and that magnetic reconnection events could occur on these kinds of cool objects. Such magnetic activity and thus the strong radio emission could be associated with the differential rotation between the atmosphere and the core of the UCD.

Berger (2002) reported Very Large Array (VLA) observations of 12 late $\mathrm{M}$ and $\mathrm{L}$ dwarfs in the solar neighborhood. Flare-like outbursts as well as persistent quiescent emission were detected from three of the 12 sources, TVLM 513-46546 (TVLM 513 hereafter), 2MASS 
J00361617+1821104 (2MASS J0036+18) and BRI 0021-0214. Among these three radio-active sources, TVLM 513 and BRI 0021, plus the first detected radio-active UCD, LP944-20, all have rapid rotational velocities, $v \sin i>30 \mathrm{~km} \mathrm{~s}^{-1}$. Hallinan et al. (2006) presented observations of the rapidly rotating M9 dwarf TVLM 513 obtained simultaneously at 4.88 and $8.44 \mathrm{GHz}$ using the VLA. The periodic radio emission at both frequencies indicated a period of $\sim 2 \mathrm{~h}$ in excellent agreement with the estimated period of rotation of the dwarf based on its $v \sin i$ of $\sim 60 \mathrm{~km} \mathrm{~s}^{-1}$. One more radio-active source from Hallinan et al. (2008), LSR J1835+3259, also has a high rotational velocity, $v \sin i \approx 50 \mathrm{~km} \mathrm{~s}^{-1}$.

Up to now, about 10 radio active ultracool dwarfs, with spectral type from M8 to L3.5, including a binary system (2MASS J07464256+2000321, hereafter 2MASS 0746+20), have been found from various surveys (Berger 2002; Burgasser \& Putman 2005; Berger 2006; Hallinan et al. 2006, 2007, 2008; Antonova et al. 2008). Three of these have been shown to have periodic radio emission, with periods of $1.96 \mathrm{~h}$ (TVLM 513, Hallinan et al. 2007), 3.07 h (2MASS J0036+18, Hallinan et al. 2008), and $2.83 \mathrm{~h}$ (LSR 1835+32, Hallinan et al. 2008).

The L dwarf binary, 2MASS $0746+20$, reported by Antonova et al. (2008) from a mini-survey of UCDs at $4.9 \mathrm{GHz}$ has a high mean flux level of $286 \pm 24 \mu \mathrm{Jy}$. Berger et al. (2009) presented an $8.5 \mathrm{~h}$ simultaneous radio, X-ray, UV, and optical observation of this binary. The strong radio emission consists mainly of short-duration periodic pulses at $4.86 \mathrm{GHz}$ with $P=124.32 \pm 0.11 \mathrm{~min}$. The radio pulses are $1 / 4$ phase different from the $\mathrm{H} \alpha$ emission.

The narrow bunching of multiple pulses of both left- and right- $100 \%$ polarized radio emission detected from TVLM 513, which originate in regions of opposite magnetic polarity, reveal the likely presence of a dipolar component to the largescale magnetic field (Hallinan et al. 2008). Zeeman Doppler Imaging (ZDI) observations have also shown that such a largescale dipolar magnetic structure could exist on an M4 dwarf star, V374 Peg, which is also a fully convective rapid rotator similar to TVLM 513 (Donati et al. 2006; Morin et al. 2008). The topology of magnetic fields on UCDs needs to be constrained by more observations.

The radio emission composed of quiescent and pulsing components from UCDs can be associated with not only the geometry of the emitting region and rotation of UCDs, but also the behavior of plasma in the magnetic field, i.e. the radiation mechanism. The very high brightness temperature and high (up to $100 \%$ ) circular polarization of the pulses (Hallinan et al. 2007) point towards an efficient, coherent radiation mechanism, the electron cyclotron maser instability (ECMI, Melrose \& Dulk 1982). This wave magnification process of the free-space radiation modes could be induced by some kind of anisotropic velocity distributions of electrons, such as a loss-cone distribution (Lau \& Chu 1983), ring shell distribution, or horseshoe distribution (Pritchett 1984).

ECMI was successfully applied to the auroral kilometric radiation (AKR) on Earth (Wu \& Lee 1979; Ergun et al. 2000), decametric radiation (DAM) on Jupiter, Saturnian kilometric radiation (SKR) (Zarka 1998, 2004) and solar millisecond microwave spikes (Aschwanden 1990b). Various authors have suggested its presence in exoplanets (Zarka 2007; Grießmeier et al. 2007; Jardine \& Cameron 2008). The magnitude of any contribution from incoherent gyrosynchrotron or synchrotron radiation to the quiescent components in UCDs is uncertain.

The generation of ECMI emission is dependent on the environment of the emitting region, such as the magnetic field (strength, structure), the electron density distribution (number, energy), the line-of-sight source scale, and the angle between the line of sight and the magnetic field (Melrose \& Dulk 1982), and also the details (velocity space gradients) of the loss cone distribution (Aschwanden 1990a). This results in the possibility of transient radio emission. The findings of Antonova et al. (2007) indicate that UCDs may also have sporadic long-term variability in their levels of quiescent radio activity. This phenomenon could be related to the change of the environment of the radio-emitting region, leading to self-quenching of the electron-cyclotron maser. Radio observations can help us to determine the magnetic configuration and the nature of the plasma in the emitting region.

For the purpose of understanding the magnetic topology, we built an active region model based on the rotation of TVLM 513 and the ECMI mechanism to simulate the observed light curve. We summarize the previous radio observation on TVLM 513 in Sect. 2. In Sect. 3, we present the model, and results are given in Sect. 4. We discuss these results in Sect. 5 and draw conclusions in Sect. 6.

\section{Previous observations on TVLM 513}

TVLM 513 is a young radio active M8.5V dwarf with a bolometric magnitude of $\log \left(L_{\mathrm{bol}} / L_{\odot}\right) \approx-3.65$, effective temperature $T_{\mathrm{eff}} \approx 2200 \mathrm{~K}$ (Tinney et al. 1993, 1995; Leggett et al. 2001), and situated at a distance of $d=10.6$ pc (Dahn et al. 2002). From the theory of the formation and evolution of UCDs and the absence of lithium on TVLM 513, it is reasonable to infer values for the mass and radius of this star of $\sim 0.07 M_{\odot}$ and $\sim 0.1 R_{\odot}$ respectively (Reid et al. 2002; Chabrier \& Baraffe 2000).

With the VLA a highly right-circularly polarized $(\sim 65 \%)$ radio event from TVLM 513 was detected with a flux density of $\sim 1100 \mu \mathrm{Jy}$, as well as persistent variable emission at $8.46 \mathrm{GHz}$ (Berger 2002). Osten et al. (2006) conducted a multifrequency VLA observation of TVLM 513 at 8.4, 4.8 and $1.4 \mathrm{GHz}$, using a strategy that involved time-sharing a single $10 \mathrm{~h}$ observation between the various frequency bands. TVLM 513 was detected at each frequency band with only marginal confirmation of variability and no detection of flares or strong circular polarization.

Again, using the VLA, Hallinan et al. (2006) found persistent and periodic radio emission from TVLM 513 at $8.44 \mathrm{GHz}$ and $4.88 \mathrm{GHz}$ simultaneously, with a period of $\sim 2 \mathrm{~h}$. Subsequently, extremely regular periodic bursts ( $p=1.96 \mathrm{~h}$, up to $\sim 4 \mathrm{mJy}$ ) of high brightness and highly circularly polarized radio emission were reported by Hallinan et al. (2007). Multiple bursts of both left and right $100 \%$ circularly polarized emission were detected. Interestingly, the radio emission can switch states from nearly $100 \%$ left polarization to $100 \%$ right polarization in each phase.

Another radio burst with a flux density up to $\sim 4 \mathrm{mJy}$ was presented by Berger et al. (2008) from a period of simultaneous radio, X-ray, ultraviolet, and optical spectroscopic observations. Steady quiescent radio emission superposed with multiple, short-duration, highly polarized bursts was observed, but these authors reported a non-periodicity in the pulses/flaring activity. In a re-analysis of this data, plus data taken $\approx 40$ days later (June 2007), Doyle et al. (2010) reported the $1.96 \mathrm{~h}$ periodicity in both datasets, deriving a more accurate period.

\section{Model}

In this section, we present a model (Fig. 1) to simulate the observational time profile of the radio flux density from TVLM 513 by assuming ECM emission is the dominant radiation mechanism. 


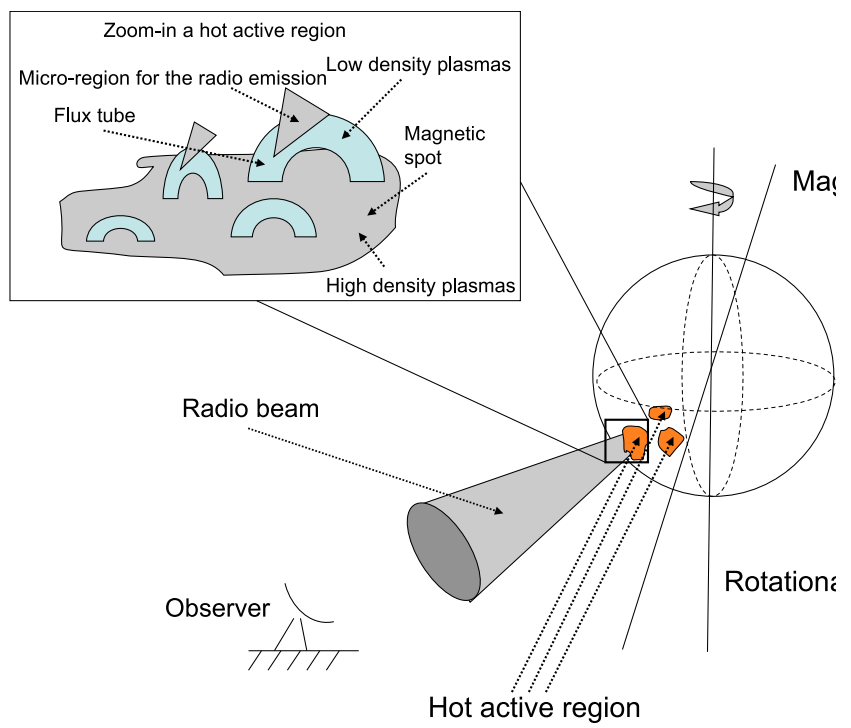

Fig. 1. A sketch of the active region model on ultracool dwarfs.

When plasma electrons are energized in magnetic flux tubes with converging legs and foot-points in a high density atmosphere (perhaps as a consequence of magnetic reconnection), some of these fast electrons collide with the high density atmosphere and thermalise at the foot-points. The remaining fast electrons are reflected in the converging field by a magnetic mirror effect. This process results in the formation of the anisotropic distribution of the plasma in velocity space, i.e. a loss-cone distribution.

The plasma including a loss-cone distribution is unstable; instability arises very quickly from such a distribution. A large amount of free energy can be released via the instability and converted to electromagnetic waves - see Dulk (1985) for a review of the process. An external magnetic-field-aligned electric field (Cattell et al. 1998; Zarka 1998; Ergun et al. 2000) induced by a time-varying external current source (Omura et al. 2003) would further modify the plasma velocity distribution to shell or horseshoe form, leading to an enhanced ECMI emission. In this paper, we assume for simplicity that the ECMI emission from UCDs is driven by the loss-cone distribution. The existence of the electric field and its effect will be addressed in future work.

In a loss-cone region where the ECMI operates, the maser radiation is concentrated on the surface of a hollow cone as discussed by Melrose \& Dulk (1982) (see Fig. 1 top panel). The half-angle $\alpha_{0}$ of the hollow cone depends on the ratio of the velocity of the plasma electrons to the speed of light, i.e. $\cos \alpha_{0}=v_{\mathrm{p}} / c$. For example, if $v_{\mathrm{p}} / c=0.5$, we have $\alpha_{0}=60^{\circ}$. The surface of the cone should be very thin with $\Delta \alpha \approx v_{\mathrm{p}} / c$. The maser emission in the loss-cone region can be observed if the line of sight is located within a thin conical sheet with thickness $\Delta \alpha$. For a low magnetic loop with a small angle between the magnetic field and the surface of the UCD, the maser emission can be seen when the emission is near the top of the loop. The maser emission from near the foot-point (where the magnetic field is almost perpendicular to the surface of UCD) can be seen when the loop is near the limb. However, for a large scale, the maser emission could have an angular distribution. In our calculation, we assume we can observe the maser emission in each flux tube in the active region.

By analogy to the solar coronal radio emission powered by two populations of plasma from the Sun with different velocity, we propose there be similar active regions on UCDs. We note that, however, an alternative mechanism, where the hot plasma beam could result from the interaction of a close-in companion of the UCD, i.e. magnetized or non-magnetized satellites resulting in auroral emission, similar to Io-Jupiter system (Queinnec \& Zarka 1998; Saur et al. 2004; Zarka et al. 2005), is possible. ECM emission has been detected in compact objects, such as white dwarfs (Willes \& Wu 2004, 2005) or neutron stars (Wolszczan \& Frail 1992). We are not able to rule out this model and the competition between the two models should be investigated in further work.

\subsection{Flux calculation}

The fine structure and time interval of the observations described in Sect. 2 indicate that the radio-emitting region on TVLM 513 would consist of complex substructures. The detected flux density $\tilde{S}$ may be the sum of several small sources

$\tilde{S}=\sum_{\mathrm{i}=0}^{\tilde{n}(t)} S_{\mathrm{i}}$.

where $S_{\mathrm{i}}$ is the flux density for a small source which can be determined by the relation (Dulk 1985)

$S_{\mathrm{i}}=\int k_{\mathrm{B}}\left(\frac{f}{c}\right)^{2} T_{\mathrm{b}} \mathrm{d} \Omega$

where $T_{\mathrm{b}}$ is the brightness temperature of a source, $k_{\mathrm{B}}$ is the Boltzmann constant, $f$ is the observed frequency, $c$ is the speed of light, $\mathrm{d} \Omega$ is the differential solid angle. If we assume that the radiation is isotropic, the differential solid angle should depend on the radius of the flux tube $r_{\text {tube }}$ of the small source and its distance from the observer. The flux density $S_{i}$ can be expressed as

$S_{\mathrm{i}}=k_{\mathrm{B}}\left(\frac{f}{c}\right)^{2} T_{\mathrm{b}} \frac{\pi r_{\text {tube }}^{2}}{4 \pi d^{2}}$.

For simplicity, we assume that the active region has a symmetric shape, and the small sources are randomly distributed within the region. The number of small sources $\tilde{n}(t)$ may be determined by rotation of the UCD. We set the time $t=0$ to be the moment when the first active region emerges in the field of view of the observer; this is also the onset time of a radio pulse. With the rotation of the $\mathrm{UCD}$, the area of the active region seen by the observer increases until it reaches a maximum, and then it decreases and disappears from the field of view of the observer. The maximum number of small sources is

$\tilde{n}_{\max } \approx\left(\frac{\Delta t \cdot 2 \pi R^{\prime} \cos \theta / T_{\mathrm{UCD}}}{r_{\text {tube }}}\right)^{\gamma}$.

where $T_{\mathrm{UCD}}$ is the rotation period, $R^{\prime}$ is the height of the active region, $\theta$ is the latitude of the active region and $\Delta t$ is the time interval from the beginning of a pulse to the maximum flux. The time duration of a pulse would be $2 \Delta t$ for a symmetric shape. We assume that the radio emission is from a thin shell of the active region near the surface of the UCD, so that we can take $\gamma \approx 2$ and $R^{\prime} \approx R_{\mathrm{UCD}}$ where $R_{\mathrm{UCD}}$ is the radius of the $\mathrm{UCD}$.

\subsection{Brightness temperature and relative parameters}

The brightness temperature of small sources depends strongly on the growth rate of the ECM emission and incoherent radiation of the background plasma. If we assume the maser emission is operated by the loss-cone distribution of a population of 
Table 1. Parameters to determine the flux density.

\begin{tabular}{lccccc}
\hline \hline Plasma & $\begin{array}{c}n_{\mathrm{h}} \\
\left(\mathrm{cm}^{-3}\right)\end{array}$ & $\begin{array}{c}T_{\mathrm{h}} \\
(\mathrm{K})\end{array}$ & $u\left(f_{\mathrm{p}} / f_{\mathrm{c}}\right)$ & $\begin{array}{c}T_{\mathrm{c}} \\
(\mathrm{K})\end{array}$ & $\begin{array}{c}T_{\mathrm{w}} \\
(\mathrm{K})\end{array}$ \\
& $1.25 \times 10^{5}$ & $10^{7}$ & 0.1 & $10^{6}$ & $10^{14}$ \\
\hline Loss-cone & $\alpha_{\mathrm{c}}$ & $N$ & $B$ & & \\
& $($ degree $)$ & & $(\mathrm{G})$ & & \\
& 30 & 6 & 1750 & & \\
\hline UCD & $R_{\mathrm{UCD}}$ & $T_{\mathrm{UCD}}$ & $r_{\text {tube }}$ & $\theta$ & $A$ \\
& $(\mathrm{~km})$ & $(\mathrm{h})$ & $(\mathrm{km})$ & $($ degree $)$ & $\left(\mathrm{km}^{2}\right)$ \\
& $7.1 \times 10^{4}$ & 1.96 & 55 & 30 & $820^{2}$ \\
\hline
\end{tabular}

Notes. Plasma parameters: $n_{\mathrm{h}}=$ density of hot plasma, $T_{\mathrm{h}}=$ temperature of hot plasma, $u=f_{\mathrm{p}} / f_{\mathrm{c}}=$ ratio of plasma frequency and gyrocyclotron frequency, $n_{\mathrm{c}}=$ density of cold (background) plasma, $T_{\mathrm{c}}=$ temperature of cold (background) plasma, $T_{\mathrm{w}}=$ background wave energy; Loss cone parameters: $\alpha_{\mathrm{c}}=$ loss cone angle, $N=$ loss cone pitch angle distribution slope, $B=$ magnetic field strength; UCD parameters: $R_{\mathrm{UCD}}=$ radius of UCD, $T_{\mathrm{UCD}}=$ rotation period of $\mathrm{UCD}, r_{\text {tube }}=$ radius of flux tube, $\theta=$ latitude of radio-emitting region, $A=$ size of emitting region. Note that the number density of cold plasma is associated with $f_{\mathrm{p}} / f_{\mathrm{c}}$.

hot plasma expressed by a Maxwellian multiplied by the function $\sin ^{N}\left(\alpha / \alpha_{\mathrm{c}} \cdot \pi / 2\right)$ and an isotropic Maxwellian distribution of a cold background plasma, the brightness temperature can be determined by the parameters of these two types of plasma. We adopt the quasi-linear theory developed by Aschwanden (1990a) to determine the growth rate and the efficiency of energy conversion. Here, we summarize the basic assumptions and the effects of several free parameters.

The quasi-linear code of Aschwanden (1990b) describes the evolution of the ECM instability and the wave-particle interactions by solving the kinetic wave-particle equations in a locally homogeneous plasma. The wave equation includes induced gyroresonance emission/absorption (for the $\mathrm{X}-, \mathrm{O}-, \mathrm{Z}-$ magneto-ionic modes and whistlers), but neglects spontaneous emission, free-free absorption, collisional deflection, and spatial wave propagation. The coupled diffusion equation contains the quasi-linear diffusion coefficients due to maser growth/damping, but neglects slower processes like particle loss and source terms. This assumption corresponds to the strong diffusion case. The quasi-linear diffusion process of the ECM instability in the solar corona successfully accounted for the time profile and observational characteristics of decimetric millisecond spikes (Aschwanden 1990b).

In order to constrain the growth rate $\Gamma$, energy conversion factor $\varepsilon_{\mathrm{c}}$ and saturation of the maser, we need to know the initial nature of the two populations of plasma and the shape of the loss-cone, i.e. for the hot plasma: particle density $n_{\mathrm{h}}$, particle temperature $T_{\mathrm{h}}$; for the cold plasma: particle density $n_{\mathrm{c}}$, particle temperature $T_{\mathrm{c}}$; for the loss-cone: loss-cone angle $\alpha_{\mathrm{c}}$, pitch angle distribution slope $N$. Rather than choowing values of the cold plasma density $n_{\mathrm{c}}$ we fix its value via adoption of a value for $u=$ $f_{\mathrm{p}} / f_{\mathrm{c}}$, the ratio of plasma frequency $\left(f_{\mathrm{p}} \simeq 9 \times 10^{-3}\left(n_{\mathrm{c}}\right)^{1 / 2} \mathrm{MHz}\right)$ and gyrofrequency $\left(f_{\mathrm{c}} \simeq 2.86 \times B \mathrm{MHz}\right)$ where $B$ is the magnetic field strength in $\mathrm{G}$.

In the quasi-linear diffusion process, the existence of background electromagnetic wave energy $T_{\mathrm{w}}$ is taken into account. As discussed by Aschwanden (1990a) in the case of solar millisecond spikes, the initial brightness temperature could be as low as the level of thermal bremsstrahlung in the range of $10^{6} \sim$ $10^{8} \mathrm{~K}$. However, because of gyroresonance or gyrosynchrotron radiation, an enhanced photon level could exist in a flaring loop which would affect the ECM process significantly, yielding a wave turbulence at the level of $\sim 10^{15} \mathrm{~K}$. We apply $T_{\mathrm{w}}$ in the range of $10^{6}-10^{16} \mathrm{~K}$ in our model.

The free energy of the plasma with a loss-cone distribution can be converted into an equivalent electromagnetic energy. The energy conversion factor $\varepsilon_{\mathrm{c}}$ can be defined as the ratio of the change of kinetic energy between the initial state and the final state of the plasma and the initial kinetic energy. In the present paper, the amount of converted energy is about $0.5 \%$, i.e. the same as in Aschwanden (1990a).

Aschwanden (1990a) investigated how the parameters influence the growth rate and brightness temperature generated by the ECM instability and gives the general formula for the key parameters as follows:

$$
\begin{aligned}
& \Gamma=6.9 \times 10^{5}\left(\frac{n_{\mathrm{h}}}{1.25 \times 10^{6} \mathrm{~cm}^{-3}}\right)\left(-1.1+1.1\left(\frac{\alpha_{\mathrm{c}}}{30^{\circ}}\right)+\left(\frac{\alpha_{\mathrm{c}}}{30^{\circ}}\right)^{-1}\right)^{-1} \\
& \times\left(0.65+0.05\left(\frac{T_{\mathrm{h}}}{10^{8} \mathrm{~K}}\right)+0.30\left(\frac{T_{\mathrm{h}}}{10^{8} \mathrm{~K}}\right)^{-1.5}\right)^{-1} \\
& \times\left(1.15\left(\frac{N}{6}\right)-0.15\right)^{0.45} \\
& \times \begin{cases}\left(2.0-1.2\left(\frac{u}{0.1}\right)+0.2\left(\frac{u}{0.1}\right)^{2}\right), & 0.1<u \leqslant 0.24 \\
0.19\left(1.0092-0.0092\left(\frac{u}{0.1}\right)^{2}\right), & 0.24<u \leqslant 1.0 \\
0.021\left(-1.416+0.440\left(\frac{u}{0.1}\right)-0.024\left(\frac{u}{0.1}\right)^{2}\right), & 1.0<u<1.4\end{cases} \\
& T_{\mathrm{b}}=9.0 \times 10^{17}\left(\frac{n_{\mathrm{h}}}{1.25 \times 10^{6} \mathrm{~cm}^{-3}}\right)^{1.10}\left(1.4-0.4\left(\frac{\alpha_{\mathrm{c}}}{30^{\circ}}\right)^{-1}\right) \\
& \times\left(\frac{T_{\mathrm{h}}}{10^{8} \mathrm{~K}}\right)^{1.2}\left(2.0\left(\frac{N}{6}\right)^{0.3}-1.0\right)\left(\frac{\log T_{\mathrm{w}}}{14}\right)^{-0.05} \\
& \times \begin{cases}1, & 0.1<u \leqslant 0.24 \\
9, & 0.24<u \leqslant 1.0 \\
1400\left(\frac{u}{0.1}\right)^{-3}, & 1.0<u<1.4\end{cases}
\end{aligned}
$$

We plot in Fig. 2 the effect of various parameters on the growth rate and brightness temperature.

\section{Results and comparison with observations}

Many parameters, not only those describing the nature of the plasma but also those associated with the properties of the UCD, can affect the observable flux density significantly. In this section, we will discuss these parameters and compare our simulations with observations. In order to study the influence of the various physical quantities we adopt "standard" values for each parameter, listed in Table 1. Unless otherwise noted, the simulations are always for magneto-ionic $X$-mode $\sigma=-1$, harmonic number $s=1$.

\subsection{The effect of rotation}

In order to see the effect of rotation of the UCD, we first start a set of simulations by fixing the initial plasma and loss-cone parameters as listed in Table 1. In addition, as suggested by Chabrier \& Baraffe $(2000)$, we adopt the radius $\left(R_{\mathrm{UCD}}=0.1 R_{\odot}\right)$ as a constant in our simulations. We initially assume that the radio-emitting region is close to the equator (latitude $\theta=30^{\circ}$ ).

Given the above parameters, the size $(A)$ of the emitting region and the tube size of a small radio area, we show in Fig. 3 the 
S. Yu et al.: Modelling the radio pulses of an ultracool dwarf
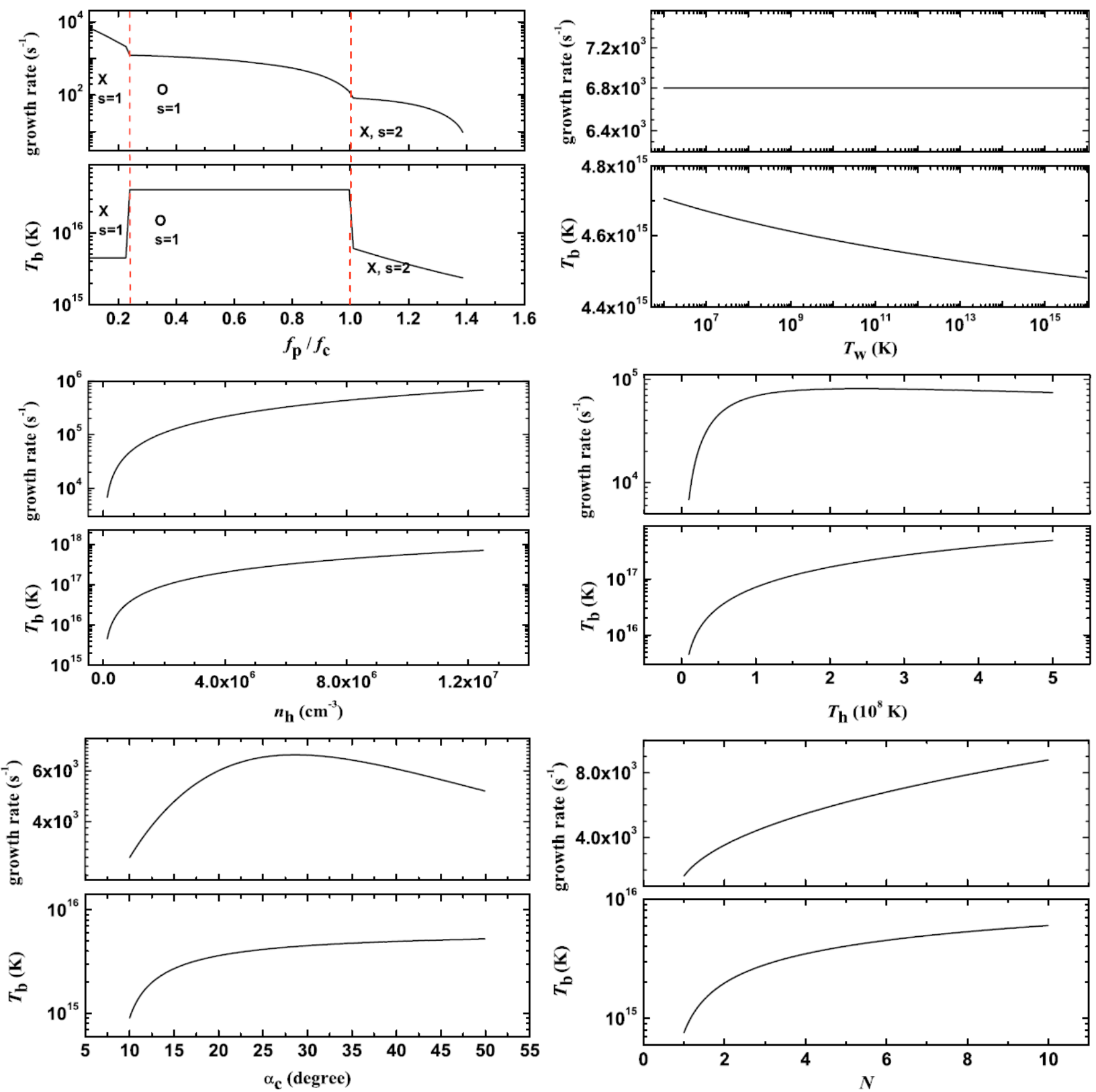

Fig. 2. The change of growth rate and brightness temperature with different plasma parameters and loss cone parameters. $f_{\mathrm{p}} / f_{\mathrm{c}}=$ ratio of plasma frequency and gyrocyclotron frequency, $T_{\mathrm{w}}=$ background wave energy, $n_{\mathrm{h}}=$ density of hot plasma, $T_{\mathrm{h}}=$ temperature of hot plasma, $\alpha_{\mathrm{c}}=$ loss cone angle, $N=$ loss cone pitch angle distribution slope, $T_{\mathrm{b}}=$ brightness temperature. The range of each parameter is in the definition of Aschwanden (1990a). We plot each panel assuming "standard" values of the other parameters (see Table 1).

influence of rotation. From the figure, we can see that the radio light curve is broadened with increasing rotation period $\left(T_{\mathrm{UCD}}\right)$ while the intensity does not change at all. This is because the intensity of the radio emission is only related to the behavior of plasma and the total size of the emitting region.

Figure 4 shows the effect of the total size $(A)$ of the radioemitting region (left panel) and the radius ( $\left.r_{\text {tube }}\right)$ of the flux tube (right panel). It is easy to understand that $r_{\text {tube }}$ cannot change the radio flux as much as $T_{\mathrm{UCD}}$. It could, however, be a controlling factor for the observed oscillation of radio flux because each flux tube could have a different environment so that we may have to give a distribution for the initial plasma parameters (see Sect. 4.5).

$A$ is an important parameter for the nature of the radioemitting region on the UCD, since we can easily see from Fig. 4 that increasing its value can lead to a rise in both intensity and pulse duration. A can be constrained by the observations once we know the rotation period of the UCD. We are able to evaluate $A$ from

$$
A \sim\left(\Delta t \cdot 2 \pi R_{\mathrm{UCD}} \cos \theta / T_{\mathrm{UCD}}\right)^{\gamma}
$$

where the parameters are the same as in Eq. (4). For the standard parameters and $\gamma=2$, we find $\Delta t \approx 15 \mathrm{~s}$. This implies that the observed $\Delta t$ can be used to estimate $A$.

\subsection{The effect of hot plasma in the loss-cone}

Because of the conservation of energy, the electromagnetic wave energy escaping from the radio- emitting region has come from the kinetic energy of the hot plasma. So the brightness temperature of one flux tube should be proportional to the number density and temperature of hot particles. 


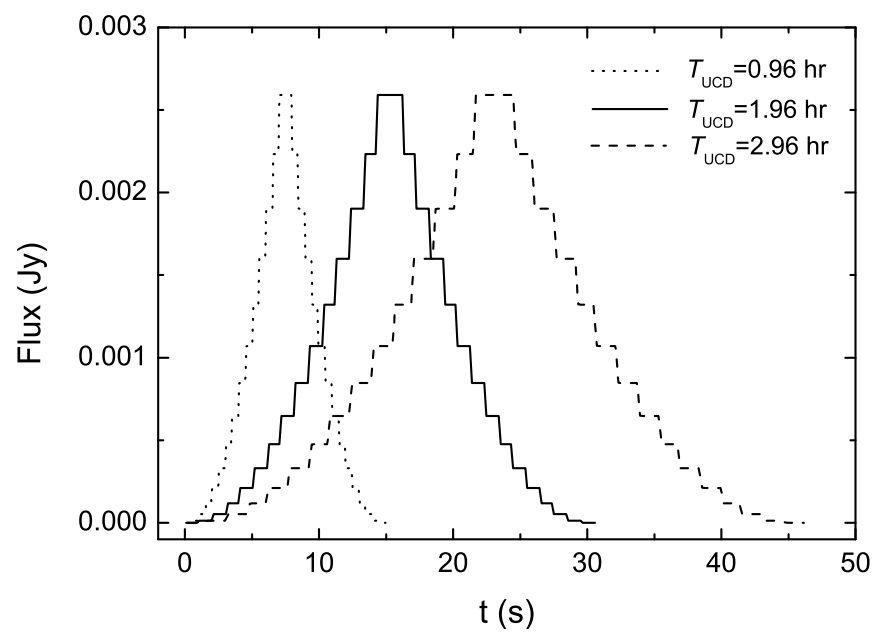

Fig. 3. The influence of rotation period $\left(T_{\mathrm{UCD}}\right)$ on the radio light curve. Vertical scale denotes the flux density while the horizontal scale indicates the time. Dashed line, solid line and dotted line are for $T_{\mathrm{UCD}} 2.96$, $1.96,0.96 \mathrm{~h}$, respectively.

We can see in panels (c) and (d) in Fig. 5 the variation of the flux density $(S)$ with the number density $\left(n_{\mathrm{h}}\right)$ and temperature $\left(T_{\mathrm{h}}\right)$ of the hot plasma. Simulations in this section assume standard values for all parameters except $n_{\mathrm{h}}$ and $T_{\mathrm{h}}$. Increasing $T_{\mathrm{h}}$ from $10^{7}$ to $10^{8} \mathrm{~K}, S$ will increase by $\sim 16$ times, while $S$ rises by a factor of 12 when $n_{\mathrm{h}}$ is one order of magnitude higher. This is consistent with the change in the brightness temperature (see Fig. 2).

It is easy to understand that the population of hot electrons is shifted to higher velocities where the number of undamped resonance ellipses increases and thus the growth rate increases with increase in temperature. On the other hand the cold background plasma will negate the loss cone and the growth rate will decrease sharply due to the lack of undamped resonance ellipses, if the hot electron temperature approaches that of the cold plasma. Melrose et al. (1984) gives a criterion for effective cyclotron damping by background cold electrons, which is $T_{\mathrm{h}} / T_{\mathrm{c}}<10 \sim 20$. This value was confirmed in the work of Aschwanden (1990a).

\subsection{The effect of cold plasma and the initial wave energy}

The number density $n_{\mathrm{c}}$ of the cold plasma can be obtained from the ratio of the plasma frequency to the cyclotron frequency $\left(f_{\mathrm{p}} / f_{\mathrm{c}}\right)$. Given a magnetic field strength $(B=1750 \mathrm{G}), f_{\mathrm{c}}=$ $4.9 \mathrm{GHz}$. Since the standard value of $f_{\mathrm{p}} / f_{\mathrm{c}}=0.1, f_{\mathrm{p}}=490 \mathrm{MHz}$. This implies $n_{\mathrm{c}}=3 \times 10^{9} \mathrm{~cm}^{-3}$, the standard value adopted in our simulations. Note from Fig. 2 , that changing $f_{\mathrm{p}} / f_{\mathrm{c}}$ from say 0.1 to 0.2 makes no difference to the brightness temperature.

In panel (a) of Fig. 5 we show the effect of changing $f_{\mathrm{p}} / f_{\mathrm{c}}$ for a constant $B$. We see that the flux density goes up a little from $f_{\mathrm{p}} / f_{\mathrm{c}}=0.23$ to $0.5\left(n_{\mathrm{c}}=1.6 \times 10^{10}\right.$ to $\left.7.4 \times 10^{10} \mathrm{~cm}^{-3}\right)$, and then drops when $f_{\mathrm{p}} / f_{\mathrm{c}}$ is increased further to $1.2\left(n_{\mathrm{c}}=4.3 \times\right.$ $10^{11} \mathrm{~cm}^{-3}$ ). This result is consistent with the variation of the brightness temperature in Fig. 2 because the flux density in our simulation depends strongly on the brightness temperature.

We note, however, that, although the growth rate declines persistently in Fig. 2, the brightness temperature increases steeply by almost one order of magnitude when the magnetoionic mode changes from $X$-mode to $O$-mode at harmonic number $s=1$. This is because of the Doppler resonance condition as discussed by Aschwanden (1990a). The resonance ellipses covering the unstable portion of the loss-cone distribution form a smaller region of positive growth for the $O$-mode, and a higher wave level results from the same amount of energy conversion.

We need to mention that only the dominant magneto-ionic modes, i.e. fundamental $(s=1) X$-mode when $f_{\mathrm{p}} / f_{\mathrm{c}}<0.24$, fundamental $(s=1) O$-mode when $0.24<f_{\mathrm{p}} / f_{\mathrm{c}}<1.0$, and second harmonic $(s=2) X$-mode when $1.0<f_{\mathrm{p}} / f_{\mathrm{c}}<1.4$, have been taken into account in our simulations. When $f_{\mathrm{p}} / f_{\mathrm{c}}>1.4$, some electrostatic instabilities become important rather than the ECM instability, and a condition for ECM emission escaping from plasma is $f_{\mathrm{p}} / f_{\mathrm{c}} \ll 1$. The fundamental $(s=1) Z$-mode may be dominant when $f_{\mathrm{p}} / f_{\mathrm{c}} \approx 0.3$ and $f_{\mathrm{p}} / f_{\mathrm{c}} \approx 1.1$ (Melrose et al. 1984; Aschwanden 1990a). However, we omit this mode because its appearance depends strongly on the ratio $f_{\mathrm{p}} / f_{\mathrm{c}}$ and another mechanism would be needed to convert it to electromagnetic radiation.

When $T_{\mathrm{w}}$ (defined as the initial wave energy) results mainly from thermal bremsstrahlung it takes a low value of $10^{6} \sim 10^{8} \mathrm{~K}$. $T_{\mathrm{w}}$ may become larger, in the range of $10^{10} \sim 10^{16} \mathrm{~K}$, due to incoherent gyroresonance or gyrosynchrotron radiation. Lower $T_{\mathrm{w}}$ can lead to a higher energy conversion efficiency because of the effect on the resonance ellipses. The flux density drops slightly with increasing $T_{\mathrm{w}}$ as seen in panel (b) of Fig. 5 .

\subsection{The effect of loss-cone parameters}

Panel (e) in Fig. 5 shows the effect of the loss-cone angle on flux density. The rapid increase of flux density when the losscone angle $\left(\alpha_{\mathrm{c}}\right)$ changes from $10^{\circ}$ to $20^{\circ}$ is the consequence of the significant increase in the size of the region of velocity space involved. Quasi-linear diffusion influences a large number of particles and results in growth and amplification of some wave modes growing. On the other hand, when $\alpha_{\mathrm{c}}>20^{\circ}$, the positive gradient in the perpendicular direction decreases so that the electromagnetic wave energy density can not be amplified effectively.

The effect of pitch angle distribution slope is similar to that of the loss-cone angle, see panel (f) in Fig. 5.

\subsection{Determining the environment of the radio-emitting region}

In this section, we determine the possible range of the different parameters. From Table 1, there are at least 13 parameters that can influence the radio light curve of TVLM 513; we are able to reduce this number, however.

Considering the contribution of the degenerate electron gas and the ionic Coulomb pressure, the radius is almost a constant around $0.1 R_{\odot}$, in the range of $0.08 R_{\odot}$ to $0.11 R_{\odot}$ (Chabrier \& Baraffe 2000).

The temperature of cold plasma is more difficult to determine. In this work we assume it is around $10^{6} \mathrm{~K}$, similar to the typical temperature of the solar corona. On the other hand, if the coronal temperature was lower, this would favor wave propagation rather than damping. We set the initial wave energy $T_{\mathrm{w}}$ at a high level of $10^{14} \mathrm{~K}$. On the other hand, from Fig. 5, the flux density varies only slightly (a factor of 2) when $T_{\mathrm{w}}$ changes from $10^{10}$ to $10^{15} \mathrm{~K}$. We should however note that Hallinan et al. (2008) suggested that the ECM instability may be a viable source of quiescent unpolarized radio emission, indistinguishable in temporal and polarization characteristics from gyrosynchrotron radiation. 
S. Yu et al.: Modelling the radio pulses of an ultracool dwarf
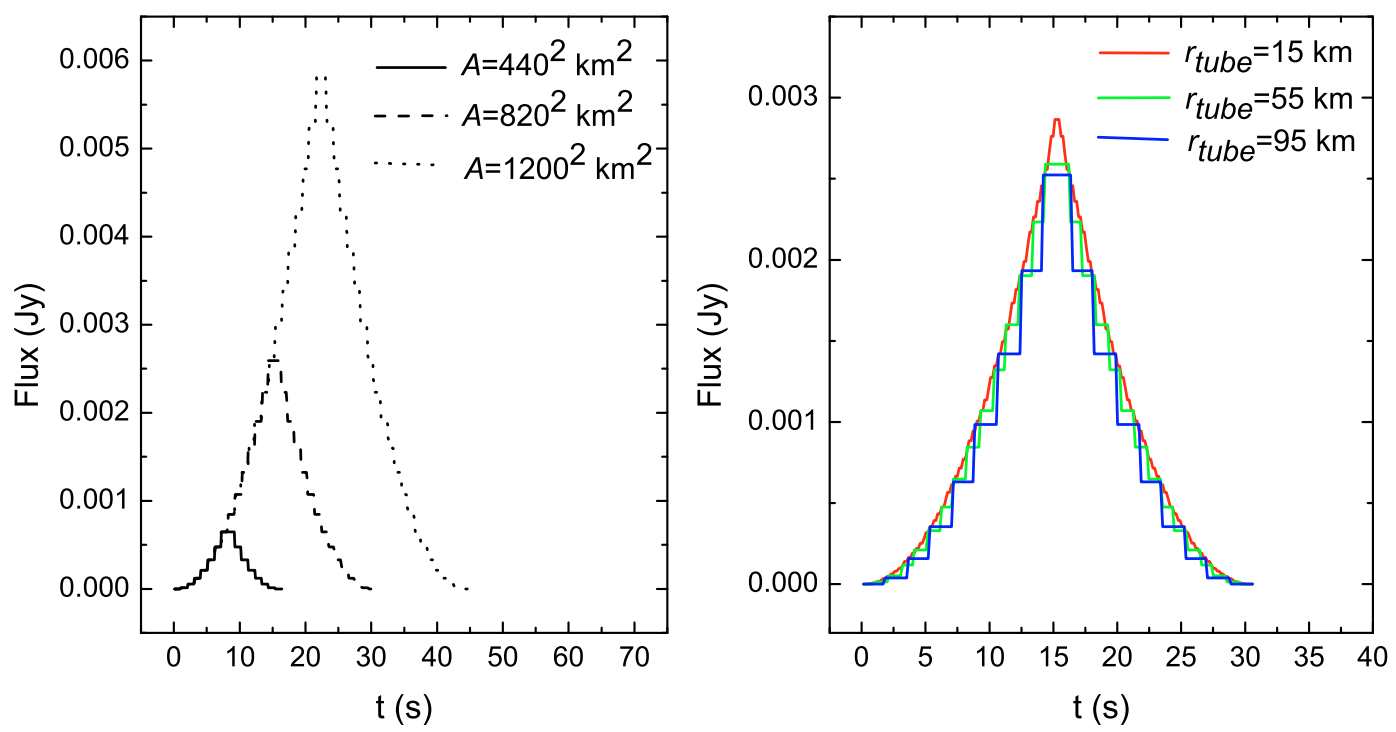

Fig. 4. Flux density against time with different size of the emission region $\left(A\right.$, left panel) and radius $\left(r_{\text {tube }}\right.$, right panel $)$ of one flux tube.
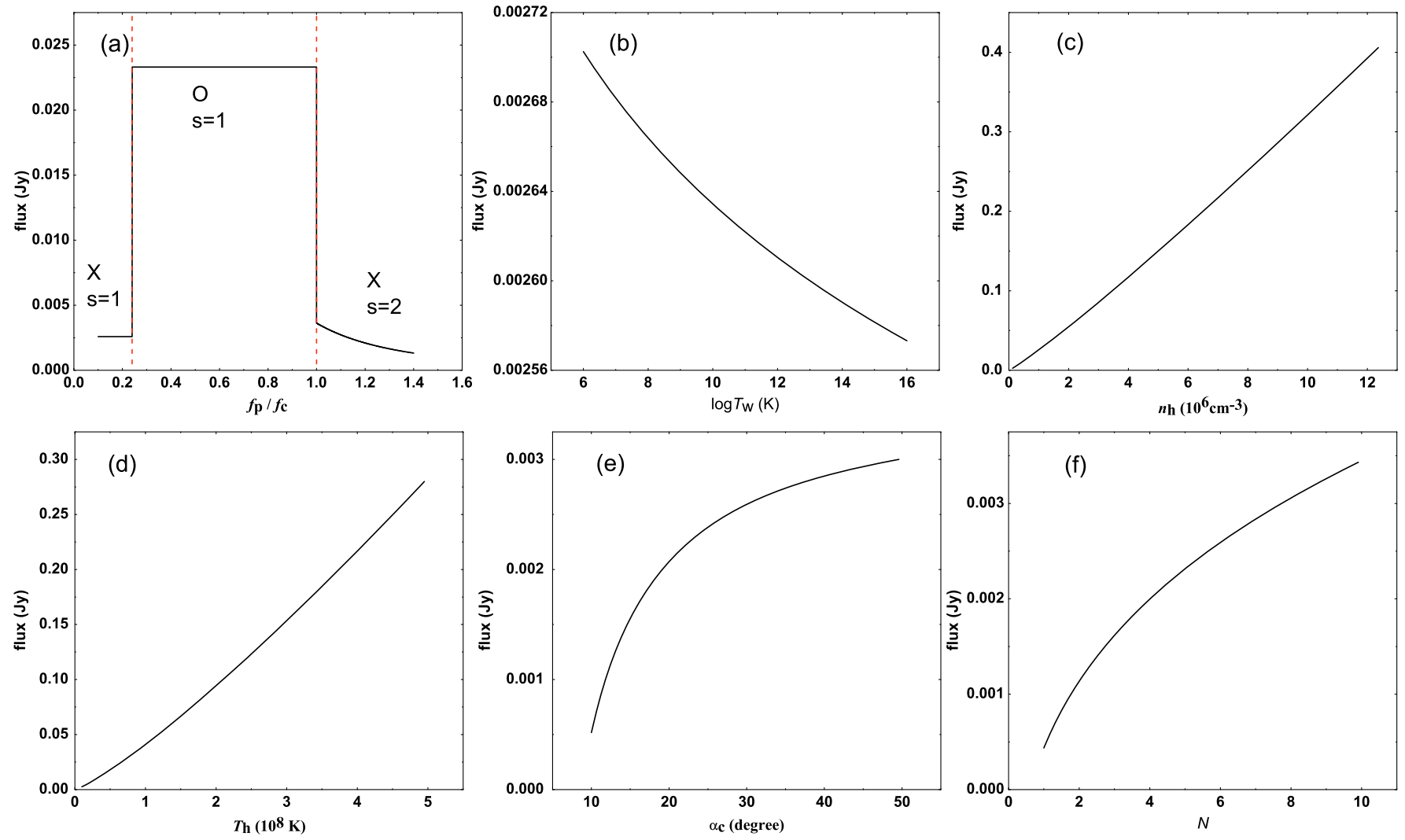

Fig. 5. Simulated maximum flux density of a radio pulse as a function of the ratio of cyclotron frequency and plasma frequency $\left(f_{\mathrm{p}} / f_{\mathrm{c}}\right)$, background wave energy $\left(T_{\mathrm{w}}\right)$, density of hot plasma $\left(n_{\mathrm{h}}\right)$, temperature of hot plasma $\left(T_{\mathrm{h}}\right)$, loss cone angle $\left(\alpha_{\mathrm{c}}\right)$, loss cone pitch angle distribution slope $(N)$ are shown in panel a) to f) respectively. The parameters for the calculation in this plot are the same as in Fig. 2.

For convenience we adopt a plausible value of 0.1 for the ratio $u$ of plasma frequency to gyrofrequency $\left(f_{\mathrm{p}} / f_{\mathrm{c}}\right)$, a value that permits unstable growth of the ECMI. If we focus on the observation frequency of $4.9 \mathrm{GHz}$, the magnetic field strength $(B)$ has to be around $1750 \mathrm{G}$ and the density of the cold background plasma is $\sim 3 \times 10^{9} \mathrm{~cm}^{-3}$. Any change of $u$ alone will not affect the maximum flux density for the fundamental $X$-mode and fundamental $O$-mode respectively. However, we stress that if we change the magnetic field strength $(B)$, even keeping $u$ at the same value, the maximum flux density will change because it depends on the cyclotron frequency.

The size of the radio-emitting region $A$ is associated with the time duration of the radio pulses. Thus in the case of TVLM 513, we have $A \approx(54.75 \Delta t)^{\gamma}$ when the latitude of the active region is $30^{\circ}$. Changing the latitude to $70^{\circ}$ gives $A \approx(21.62 \Delta t)^{\gamma}$. $\Delta t$ is easily obtained from observations and is a more convenient parameter than $A . r_{\text {tube }}$ is a variable to describe the radius of a flux tube. We expect there are distributions for plasma parameters 


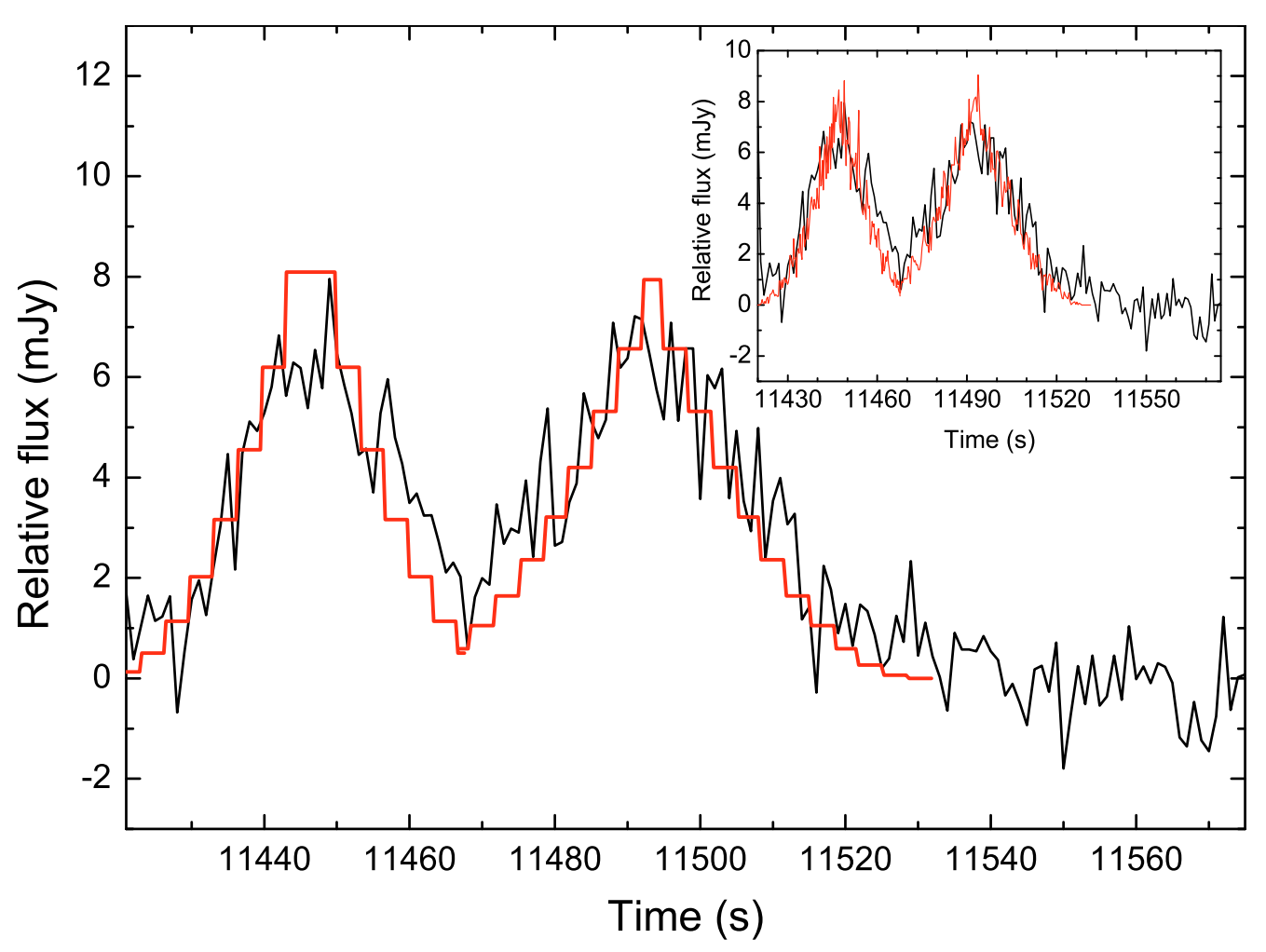

Fig. 6. Comparison of our simulation with observation taken with the Arecibo telescope by Hallinan et al. (2010). Black solid line shows the observation of 2008 May 19 (UT) at 4775 MHz. Red line shows the simulated curve. The inset panel shows the simulated light curve using a uniform distribution of the free parameters.

$\left(T_{\mathrm{h}}, n_{\mathrm{h}}\right)$ and loss-cone parameters $\left(\alpha_{\mathrm{c}}, N\right)$ in different flux tubes. The variation of different parameters can result in the oscillation of the observed flux density. Smaller values for $r_{\text {tube }}$ lead to smoother simulated radio light curves (see Fig. 4).

So now the free parameters are reduced to 6 , i.e. the density $n_{\mathrm{h}}$ and temperature $T_{\mathrm{h}}$ of hot plasma, the angle $\alpha_{\mathrm{c}}$ and pitch angle distribution slope $N$ of the loss-cone, the radius $r_{\text {tube }}$ of one flux tube and the size of the emitting region $A$. The functions of the parameters are: $n_{\mathrm{h}}$ and $T_{\mathrm{h}}$ can control the change of flux density dramatically, $\alpha_{\mathrm{c}}$ and $N$ can control the change of flux density gently, $A$ can control both flux density and time duration of the radio pulse which can be constrained by observations, and $r_{\text {tube }}$ describes the radius of the flux tube in the active region.

\subsection{Comparison with observations}

The free parameters can be constrained by making our simulations match up to observations. Figure 6 shows the comparison of our simulation with observations (see Hallinan et al. 2010, for further details). The observation is taken from 2008 May 19 (UT) at $4725 \mathrm{MHz}$ for the M8.5V dwarf TVLM 513. This means that the magnetic field strength is $1652 \mathrm{G}$ because of the cyclotron frequency. We adopt $f_{\mathrm{p}} / f_{\mathrm{c}}=0.1$, implying the density of cold plasma in the emitting region is $\sim 2.64 \times 10^{9} \mathrm{~cm}^{-3}$. The size of the radio-emitting region is constrained by the time duration of the two pulses $\left(A \approx(54.75 \Delta t)^{2}\right)$, implying $1620 \times 1620 \mathrm{~km}^{2}$ for the first pulse and $2060 \times 2060 \mathrm{~km}^{2}$ for the second pulse. The radius of each flux tube is taken as $180 \mathrm{~km}$ arbitrarily because we are not able to get any information on its value from the observed radio light curve. We take $\alpha_{\mathrm{c}}=30^{\circ}$ and $N=5$ for the first pulse, $\alpha_{\mathrm{c}}=35^{\circ}$ and $N=2$ for the second pulse. $T_{\mathrm{h}}$ and $n_{\mathrm{h}}$ are same for the both pulses, equal to $10^{7} \mathrm{~K}$ and $1.25 \times 10^{5} \mathrm{~cm}^{-3}$ respectively.

The simulated light curve reproduces the observation. However, this does not mean that the above values for the different parameters are unique. In order to compare our simulation with the observed two pulses, we take a flat distribution for $\alpha_{\mathrm{c}}$ and $N$ in the range of $10^{\circ}-50^{\circ}$ and $1-6$ for the both pulses, $n_{\mathrm{h}}:(1.25-5) \times 10^{5} \mathrm{~cm}^{-3}, T_{\mathrm{h}}:(1-4.5) \times 10^{7} \mathrm{~K}$ for the first pulse and $n_{\mathrm{h}}:(1.25-4.5) \times 10^{5} \mathrm{~cm}^{-3}, T_{\mathrm{h}}:(1-3) \times 10^{7} \mathrm{~K}$ for the second pulse (see inset panel in Fig. 6). We note that the size of the emitting region depends on the latitude $\theta$ in our simulation (see Sect. 4.5). Changing the latitude from $30^{\circ}$ (the value for the simulation) to $70^{\circ}$ gives a value for the size of $640 \times 640 \mathrm{~km}^{2}$ for the first pulse and $814 \times 814 \mathrm{~km}^{2}$ for the second pulse. Other parameters also need to change in order to fit the observed light curve.

The simulation in Fig. 6 is remarkably similar to the observations. Our results indicate that the two radio emission regions producing the two pulses are very close, which would be consistent with the nature of the cone radiation of ECMI. An important feature is that the radio pulses would repeat with the rotation period of the UCD. In the case of TVLM 513, the period is $\sim 1.96 \mathrm{~h}$. Moreover, we note that the decay time of the pulses in some observations is longer than our simulation. This is possibly due to the deformation of the radio-emitting region as fast rotation of the dwarf can cause the shape of the emitting region to vary from an almost symmetric circle to an asymmetric ellipse with a tail.

\section{Discussion}

Our results show that rotation coupled with the ECMI mechanism can account for the flux density and polarization of the radio pulses from TVLM 513 successfully. We can not exclude the possibility that the depolarization of ECMI could be due to radiation transfer of the emission in a neutral atmosphere with lower fractional ionization or that inhomogeneous dust clouds (Littlefair et al. 2008) could have an effect on the quiescent 
emission and the unpolarized components. Hallinan et al. (2006) suggested that the depolarization or mode conversion of the $X$ mode emission occurs in a density cavity, as mode conversion of terrestrial kilometric radiation (TKR) from $X$-mode to $R$-mode in the emitting density cavity (Ergun et al. 2000) may account for escape of maser emission without re-absorption at higher harmonics of the emission frequency.

For the optically thick source, i.e. optical depths $\tau \gg 1$, the brightness temperature $T_{\mathrm{b}}$ can be constrained by Eq. (3) by using the observed flux density. In order to see the relation between $T_{\mathrm{b}}$ and flux density, we rewrite the equation in the form of

$$
T_{\mathrm{b}}=5.32 \times 10^{10} \mathrm{~K}\left(\frac{S_{f}}{4 \mathrm{mJy}}\right)\left(\frac{f}{4.9 \mathrm{GHz}}\right)^{-2}\left(\frac{d}{10 \mathrm{pc}}\right)^{2}\left(\frac{R_{\mathrm{s}}}{\mathrm{R}_{\mathrm{Jup}}}\right)^{-2}
$$

where $S_{f}$ is the flux density in mJy at the frequency $f(\mathrm{GHz}), d$ the distance of the radio source from us in pc, $R_{\mathrm{S}}$ the size of the emission region in Jupiter radii $\left(1 R_{\mathrm{Jup}} \sim 0.1 R_{\odot} \approx 7 \times 10^{9} \mathrm{~cm}\right)$ (Linsky \& Gary 1983; Doyle et al. 1988; Dorman et al. 1989; Burrows et al. 1989; Leto et al. 2000). Unfortunately, when we calculate $T_{\mathrm{b}}$, we have to assume the size of the radio-emitting region. For example, observations show that the flux density of the pulses of TVLM 513 is about $4 \mathrm{mJy}$ at $\sim 4.9 \mathrm{GHz}$. Berger (2002) obtained the brightness temperature in the range of $10^{8}-10^{9} \mathrm{~K}$ by assuming the size of a corona to be $2-4 R_{\text {Jup }}$, while Hallinan et al. (2006) deduced a value of $2.9 \times 10^{10} \mathrm{~K}$ if the size of the region is $1 R_{\text {Jup }}$. In our model, $T_{\mathrm{b}} \gtrsim 5 \times 10^{10} \mathrm{~K}$ is about the temperature of the quiescent radio emission, assumed to come from a large emission region ( $\left.\lesssim 1 R_{\mathrm{Jup}}\right)$. For the radio pulses from magnetic loops, the theoretical temperature of the coherent ECMI emission can be up to $10^{15} \mathrm{~K}$, implying the emission region for the pulses is much more compact, e.g. $0.007 R_{\mathrm{Jup}}$.

The configuration and topology of magnetic field on UCDs remains unclear. In our model, we only need a simple dipole poloidal-like field to calculate the flux density and explain the high polarisation of the radio pulses. In the case of TVLM 513, multiple bursts of both left and right $100 \%$ circularly polarized emission in regions of opposite magnetic polarity indicate the existence of a dipolar large-scale magnetic field (Hallinan et al. 2007 ) or a few small active regions with scale $\ll 1 R_{\text {Jup }}$.

Another important parameter, when combined with the magnetic field strength, is the pitch angle $\beta$. Electrons with small $\beta$ less than a critical value $\beta_{\mathrm{c}}$, i.e. $\beta<\beta_{\mathrm{c}}$, precipitating into the dense atmosphere are lost, while the electrons with $\beta>\beta_{\mathrm{c}}$ will be reflected back to the flux tube to form an anisotropic velocity distribution. The value of $\beta_{\mathrm{c}}$ depends on the convergence factor which is determined by the ratio of magnetic field strengths at the top and the foot-points of the flux tube (also called magnetic mirror ratio). For a symmetric flux tube, we have (Dulk 1985)

$\beta_{\mathrm{c}}=\arcsin \left(B_{\text {top }} / B_{\text {foot }}\right)^{1 / 2}$

where $B_{\text {top }}$ and $B_{\text {foot }}$ are the magnetic field strengths at the top and in the foot-point of the magnetic flux tube, respectively. Typical values of $B_{\text {top }} / B_{\text {foot }}$ in the Sun are in the range of 0.1 to 0.5 . In the simulation, the pitch angle is approximately equal to $30^{\circ}$, which means we should have $0.5>\sin \beta_{\mathrm{c}}=$ $\left(B_{\text {top }} / B_{\text {foot }}\right)^{1 / 2}$. This could give a lower limit on $B_{\text {foot }}$.

Assuming the magnetic field is radial, the field strength of a region can be described by

$B=\frac{B_{0}}{4}\left(\frac{R}{R_{*}}-\frac{1}{2}\right)^{-2}, \quad R>R_{*}$

where $B_{0}$ is the magnetic field at the photosphere, $R$ the height of the radio emitting region measured from the centre of the star

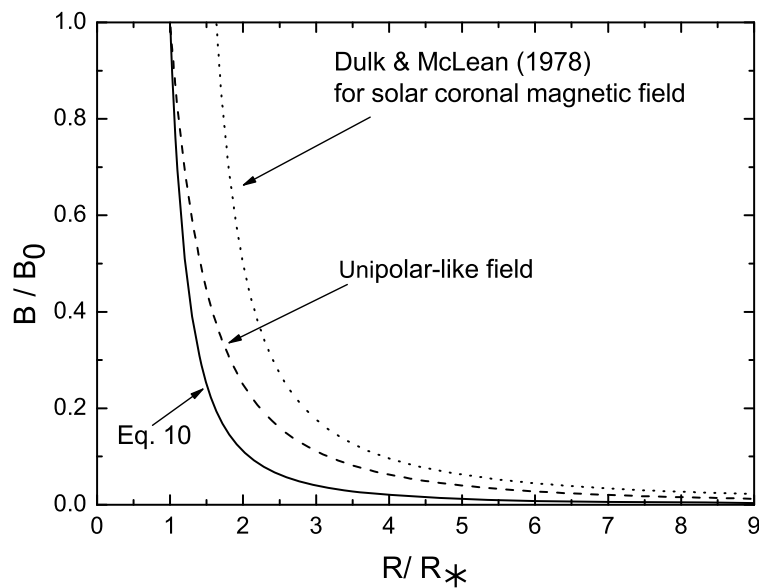

Fig. 7. Possible magnetic field structures for UCDs. The solid line is for Eq. (10) which was suggested for a dM star, the dashed line for a unipolar-like field, the dotted line for the possible field in the solar coronal region.

and $R_{*}$ the photospheric radius. This was chosen as a compromise between a unipolar-like field $B=B_{0}\left(R / R_{*}\right)^{-2}$ and the bestfit form for solar coronal magnetic fields above active regions $B=0.5\left[\left(R / R_{*}\right)-1\right]^{-1.5}$ given by Dulk \& McLean (1978) for the range $1.01<R / R_{*}<10$. This choice makes the field fall off more quickly than either a unipolar magnetic field configuration or that of the solar active region magnetic field (Gary \& Linsky 1981). Figure 7 shows the dependence of magnetic field strength on height of the radio emission region above the photosphere.

Combining Eqs. (9) and (10), the radio emission region and therefore the magnetosphere can be constrained in the range of $0.56 R_{*}$ to $6.75 R_{*}$. A possible position for the radio-emitting region in the radio active UCDs at $\sim 4.9 \mathrm{GHz}$ is $1.5 R_{*}$ from their center, $1.06 \times 10^{5} \mathrm{~km}$ in the case of TVLM 513. This implies that the magnetic field strength at the photosphere or chromosphere would be as large as $7000 \mathrm{G}$. More observations in the optical (Zeeman Doppler effect) and infrared bands are needed to constrain the magnetic topology and the behavior of the plasma in the magnetic flux tube and to determine whether such large field strengths exist on these objects.

In addition, we expect the existence of an enhanced ambient wave energy background by gyroresonance turbulence or gyrosynchoroton radiation and some intense events at other wavelengths, e.g. optical or X-ray emission, which would occur from the process where hot plasma starting from collision-less region collides with the collisional chromosphere or photosphere. In the case of X-ray emission, thermal bremsstrahlung emission and inverse Compton scattering could be the responsible mechanism since there are hot plasmas with $T_{\mathrm{h}}$ up to $10^{8} \mathrm{~K}$ and possible low energy photons. However, since the Thomson scattering optical depth of the corona of the brown dwarf is $\ll 1$, the contribution of inverse Compton scattering is unlikely to be important.

For the thermal bremsstrahlung emission of ionized hydrogen and helium dominated source, the detected flux density integrated over frequency is $S_{\mathrm{X}-\mathrm{B}}=4.67 \times$ $10^{-28} T_{\mathrm{h}}^{1 / 2} n_{\mathrm{h}} n_{\mathrm{c}} Z^{2} \bar{g}_{\mathrm{B}} R_{\mathrm{S}}^{3} d^{-2}$, where $Z$ is an ion of charge in units $e$ (here we take $Z=1$ ), $\bar{g}_{\mathrm{B}}$ the Gaunt factor (we take $\bar{g}_{\mathrm{B}}=1.2$, which gives an accuracy of $\$ 20 \%$ since $\left.1.1<\bar{g}_{\mathrm{B}}<1.5\right), R_{\mathrm{S}}$ the radius of the source, $d$ distance of the source from us and all quantities are in $c g s$ units (Rybicki \& Lightman 1979). In the case of TVLM 513, assuming that the X-ray emission comes from the same region as the radio emission, we can take the parameters as $T_{\mathrm{h}}=10^{7} \mathrm{~K}, n_{\mathrm{h}}=10^{8} \mathrm{~cm}^{-3}, n_{\mathrm{c}}=3 \times 10^{9} \mathrm{~cm}^{-3}$, $R_{\mathrm{s}}=2 \times 10^{8} \mathrm{~cm}, d=10 \mathrm{pc} \approx 3.1 \times 10^{19} \mathrm{~cm}$, hence we get the 
$S_{\mathrm{X}-\mathrm{B}}=4.42 \times 10^{-21} \mathrm{erg} \mathrm{cm}^{-2} \mathrm{~s}^{-1}$, giving an X-ray luminosity of $L_{\mathrm{X}-\mathrm{B}} \approx 5.34 \times 10^{19} \mathrm{erg} \mathrm{s}^{-1}$. The X-ray flux density/luminosity could be underestimated significantly as the $\mathrm{X}$-ray emission might be diffuse and from a larger region (perhaps $\sim 10$ times) than that of the radio emission, as is the case for the X-ray emission observed from Jupiter by S uzaku (Ezoe et al. 2010).

Interestingly, Berger et al. (2008) obtained a marginal detection in X-rays suggesting a flux density of $6.3 \times$ $10^{-16} \mathrm{erg} \mathrm{cm}^{-2} \mathrm{~s}^{-1}$ (luminosity $L_{\mathrm{X}}=8.5 \times 10^{24} \mathrm{erg} \mathrm{s}^{-1}$ ) with mean energy at $0.9 \mathrm{keV}$. This means the temperature of the hot plasma could be slightly lower than $10^{7} \mathrm{~K}$. Further multiwavelength observations will help to refine our model and its parameters to understand the radio and X-ray emission from these kinds of cool objects.

\section{Conclusions}

An active region model is applied to the radio emission from a cool dwarf, in which the ECMI mechanism is responsible for the radio bursts from the magnetic tubes, while the rotation of the dwarf can modulate the total observed flux with respect to time. The time profile of the radio light curve is in the form of power law in our model. Using this model, we can determine the nature (e.g. size, temperature, density) of the radio-emitting region plus the magnetic topology can be constrained as well.

In the case of TVLM 513, our model shows the loss-cone electrons have a density in the range of $1.25 \times 10^{5}-5 \times 10^{5} \mathrm{~cm}^{-3}$ and temperature between $10^{7}$ and $5 \times 10^{7} \mathrm{~K}$. The brightness temperature is typically $\sim 10^{15} \mathrm{~K}$ for pulses, $\sim 5 \times 10^{10} \mathrm{~K}$ for the background emission, implying the ECMI mechanism operates in compact region of $\sim 0.007 R_{\text {Jup }}$ if the active region is at $30^{\circ}$. For an active region closer to the pole, e.g. $70^{\circ}$, the size is $\sim 60 \%$ smaller, implying a higher brightness temperature.

The model predicts an enhanced ambient wave energy background and $\mathrm{a} \approx 7000 \mathrm{G}$ surface magnetic field strength. The theoretical X-ray flux density in our model is much smaller than a marginal X-ray observation of TVLM 513, which implies a more complicated plasma behavior or magnetic structure on the dwarf. Additional multi-wavelength observations are needed to constrain the tentative conclusions and help us to improve the understanding of the magnetic field on ultracool dwarfs and to test the viability of this model in comparison with others, such as the auroral model.

Acknowledgements. The Armagh Observatory is supported by a grant from the Northern Ireland Dept. of Culture Arts and Leisure. G.H. and A.G. gratefully acknowledge the support of Science Foundation Ireland (grant No. 07/RFP/PHYF553). A.K., S.Y.U. \& J.G.D. thank the Leverhulme Trust for support. AA gratefully acknowledges the support of the Scientific Research Fund of "St. Kl. Ohridski" University of Sofia (grant No. 80/2009 and 138/2010). A.L.M. \& S.Y.U. thank M. Aschwanden for providing the quasi-linear diffusion code of ECMI. SYU thanks Gavin Ramsay for his comments and also thanks Eamon Scullion for discussions. We also thank the UK Science and Technology Facilities Council for support via a Visitor grant. We gratefully thank the referee for his/her suggestions and comments.

\section{References}

Afram, N., Reiners, A., \& Berdyugina, S. V. 2009, ed. S. V. Berdyugina, K. N. Nagendra, \& R. Ramelli, ASP Conf. Ser., 405, 527

Antonova, A., Doyle, J. G., Hallinan, G., Bourke, S., \& Golden, A. 2008, A\&A, 487,317

Antonova, A., Doyle, J. G., Hallinan, G., Golden, A., \& Koen, C. 2007, A\&A, 472,257

Aschwanden, M. J. 1990a, A\&AS, 85, 1141

Aschwanden, M. J. 1990b, A\&A, 237, 512

Audard, M., Osten, R. A., Brown, A., et al. 2007, A\&A, 471, L63

Berger, E. 2002, ApJ, 572, 503
Berger, E. 2006, ApJ, 648, 629

Berger, E., Ball, S., Becker, K. M., et al. 2001, Nature, 410, 338 Berger, E., Gizis, J. E., Giampapa, M. S., et al. 2008, ApJ, 673, 1080 Berger, E., Rutledge, R. E., Phan-Bao, N., et al. 2009, ApJ, 695, 310 Burgasser, A. J., \& Putman, M. E. 2005, ApJ, 626, 486

Burgasser, A. J., Kirkpatrick, J. D., Reid, I. N., et al. 2000, AJ, 120, 473

Burrows, A., Hubbard, W. B., \& Lunine, J. I. 1989, ApJ, 345, 939

Cattell, C., Bergmann, R., Sigsbee, K., et al. 1998, Geophys. Res. Lett., 25, 2053

Chabrier, G., \& Baraffe, I. 2000, ARA\&A, 38, 337

Dahn, C. C., Harris, H. C., Vrba, F. J., et al. 2002, AJ, 124, 1170

Donati, J., Forveille, T., Cameron, A. C., et al. 2006, Science, 311, 633

Dorman, B., Nelson, L. A., \& Chau, W. Y. 1989, ApJ, 342, 1003

Doyle, J. G., Butler, C. J., Bryne, P. B., \& van den Oord, G. H. J. 1988, A\&A, 193, 229

Doyle, J. G., Antonova, A., Marsh, M. S., Hallinan, G. Yu, S., \& Golden, A. 2010, A\&A, 524, A15

Dulk, G. A. 1985, ARA\&A, 23, 169

Dulk, G. A., \& McLean, D. J. 1978, Sol. Phys., 57, 279

Ergun, R. E., Carlson, C. W., McFadden, J. P., et al. 2000, ApJ, 538, 456

Ezoe, Y., Ishikawa, K., Ohashi, T., et al. 2010, ApJ, 709, L178

Gary, D. E., \& Linsky, J. L. 1981, ApJ, 250, 284

Gizis, J. E., Monet, D. G., Reid, I. N., et al. 2000, AJ, 120, 1085

Grießmeier, J., Zarka, P., \& Spreeuw, H. 2007, A\&A, 475, 359

Guedel, M., \& Benz, A. O. 1993, ApJ, 405, L63

Hallinan, G., Antonova, A., Doyle, J. G., et al. 2006, ApJ, 653, 690

Hallinan, G., Bourke, S., Lane, C., et al. 2007, ApJ, 663, L25

Hallinan, G., Antonova, A., Doyle, J. G., et al. 2008, ApJ, 684, 644

Jardine, M., \& Cameron, A. C. 2008, A\&A, 490, 843

Lau, Y. Y., \& Chu, K. R. 1983, Phys. Rev. Lett., 50, 243

Leggett, S. K., Allard, F., Geballe, T. R., Hauschildt, P. H., \& Schweitzer, A. 2001, ApJ, 548, 908

Leto, G., Pagano, I., Linsky, J. L., Rodonò, M., \& Umana, G. 2000, A\&A, 359, 1035

Liebert, J., Kirkpatrick, J. D., Cruz, K. L., et al. 2003, AJ, 125, 343

Linsky, J. L., \& Gary, D. E. 1983, ApJ, 274, 776

Littlefair, S. P., Dhillon, V. S., Marsh, T. R., et al. 2008, MNRAS, 391, L88

Melrose, D. B., \& Dulk, G. A. 1982, ApJ, 259, 844

Melrose, D. B., Dulk, G. A., \& Hewitt, R. G. 1984, J. Geophys. Res., 89, 897

Mohanty, S., Basri, G., Shu, F., Allard, F., \& Chabrier, G. 2002, ApJ, 571, 469

Morin, J., Donati, J., Forveille, T., et al. 2008, MNRAS, 384, 77

Neuhäuser, R., Briceño, C., Comerón, F., et al. 1999, A\&A, 343, 883

Omura, Y., Heikkila, W. J., Umeda, T., Ninomiya, K., \& Matsumoto, H. 2003, J. Geophys. Res. (Space Physics), 108, 1197

Osten, R. A., Hawley, S. L., Bastian, T. S., \& Reid, I. N. 2006, ApJ, 637, 518

Parker, E. N. 1955, ApJ, 122, 293

Pritchett, P. L. 1984, J. Geophys. Res., 89, 8957

Queinnec, J., \& Zarka, P. 1998, J. Geophys. Res., 103, 26649

Reid, I. N., Kirkpatrick, J. D., Gizis, J. E., \& Liebert, J. 1999, ApJ, 527, L105

Reid, I. N., Kirkpatrick, J. D., Liebert, J., et al. 2002, AJ, 124, 519

Reiners, A., \& Basri, G. 2007, ApJ, 656, 1121

Reiners, A., \& Basri, G. 2010, ApJ, 710, 924

Rockenfeller, B., Bailer-Jones, C. A. L., Mundt, R., \& Ibrahimov, M. A. 2006, MNRAS, 367, 407

Rutledge, R. E., Basri, G., Martín, E. L., \& Bildsten, L. 2000, ApJ, 538, L141

Rybicki, G. B., \& Lightman, A. P. 1979, Radiat. Processes Astrophys. (New York: Wiley-Interscience), 393

Saur, J., Neubauer, F. M., Connerney, J. E. P., Zarka, P., \& Kivelson, M. G. 2004, Plasma interaction of Io with its plasma torus, ed. Bagenal, F., Dowling, T. E., \& McKinnon, W. B., 537

Schmidt, S. J., Cruz, K. L., Bongiorno, B. J., Liebert, J., \& Reid, I. N. 2007, AJ, 133,2258

Stelzer, B., Micela, G., Flaccomio, E., Neuhäuser, R., \& Jayawardhana, R. 2006a, A\&A, 448, 293

Stelzer, B., Schmitt, J. H. M. M., Micela, G., \& Liefke, C. 2006b, A\&A, 460, L35

Tinney, C. G., Mould, J. R., \& Reid, I. N. 1993, AJ, 105, 1045

Tinney, C. G., Reid, I. N., Gizis, J., \& Mould, J. R. 1995, AJ, 110, 3014

West, A. A., Hawley, S. L., Walkowicz, L. M., et al. 2004, AJ, 128, 426

Willes, A. J., \& Wu, K. 2004, MNRAS, 348, 285

Willes, A. J., \& Wu, K. 2005, A\&A, 432, 1091

Wolszczan, A., \& Frail, D. A. 1992, Nature, 355, 145

Wu, C. S., \& Lee, L. C. 1979, ApJ, 230, 621

Zarka, P. 1998, J. Geophys. Res., 103, 20159

Zarka, P. 2004, Adv. Space Res., 33, 2045

Zarka, P. 2007, Planet. Space Sci., 55, 598

Zarka, P., Hess, S., \& Mottez, F. 2005, AGU Fall Meeting Abstracts, A1275

Article number, page 10 of 10 